\section{JTI}

JOURNAL OF

TRAUMA AND INJURY

Received: July 24, 2020

Revised: September 17, 2020

Accepted: September 21, 2020

Correspondence to

Ye Rim Chang, M.D., Ph.D.

Department of Trauma Surgery, Trauma Center, Dankook University Hostpital, 20

Manghyang-ro, Dongnam-gu, Cheonan

31116, Korea

Tel: +82-41-550-0038

Fax: +82-41-550-0039

E-mail:yrchang@dkuh.co.kr

\title{
Positioning of Resuscitative Endovas- cular Balloon Occlusion of the Aorta Catheter: A Case of an Elderly Patient with Concomitant Chest and Pelvic Injury after Blunt Trauma
}

\author{
Dongsub Noh, M.D., M.S. ${ }^{1}$, Jeongseok Yun, M.D., M.S. ${ }^{2}$, \\ Ye Rim Chang, M.D., Ph.D. ${ }^{2}$ \\ ${ }^{1}$ Department of Thoracic and Cardiovacular Surgery, Trauma Center, Dankook University \\ Hospital, Cheonan, Korea \\ ${ }^{2}$ Department of Trauma Surgery, Trauma Center, Dankook University Hospital, Cheonan, \\ Korea
}

Resuscitative endovascular balloon occlusion of the aorta (REBOA) has been increasingly used in recent years as a resuscitative adjunct for trauma patients with life-threatening non-compressible torso hemorrhage. By blocking the aorta temporarily with an inflated balloon, REBOA preserves cerebral and coronary perfusion while diminishing exsanguination below the balloon, thereby providing time for resuscitation and definitive bleeding control. When determining the occlusion zone during the REBOA procedure, factors such tortuosity of the aorta, co-occurring minor chest injuries, and the severity of shock must be considered, as well as the main injury site. This paper describes a case of high Zone I REBOA in an elderly patient with a tortuous aorta who had concomitant injuries of the chest and pelvis.

Keywords: Trauma; Balloon occlusion; Indication; Elderly; Chest injury

\section{INTRODUCTION}

Resuscitative endovascular balloon occlusion of the aorta (REBOA) has increasingly been used recently as a resuscitative adjunct for trauma patients with life-threatening non-compressible torso hemorrhage. By blocking the aorta temporarily with an in(http://creativecommons.org/licenses/by-nc/4.0/) which permits unrestricted noncommercial use, distribution, and reproduction in any medium, provided the original work is properly cited. 
flated balloon, REBOA preserves cerebral and coronary perfusion while diminishing the exsanguination below the balloon and provides time for the resuscitation and definitive bleeding control [1,2].

In general, focused assessment for trauma (FAST) and a pelvic radiograph can provide information for possible causes of hemorrhagic shock in trauma patients and be a determinant for the level of occlusion. However, factors such tortuosity of the aorta, combined minor chest injury, and profound shock of the patient have to be considered as well as the main injury site. The position at Zone I is effective for these cases; the patient who has tortuous aorta or expecting more hemodynamic effect or concomitant minor thoracic injury (highly Zone I). Herein, we report a case of high Zone I REBOA in an elderly patient with a tortuous aorta who had concomitant injuries of chest and pelvis.

\section{CASE REPORT}

A 82-year-old woman was struck by a car and transferred to the nearest hospital. She was diagnosed with unstable pelvic bone fracture (Tile's B2 class, lateral compression type), retroperitoneal hemorrhage, right multiple rib fractures with right hemothorax on the outside computed tomography (CT) scan (Fig. 1). Right closed thoracostomy was done and then the patient was transferred to the Level I trauma center. On arrival, three hours had been passed since the accident and $80 \mathrm{cc}$ of blood had been drained from closed thoracostomy. The initial vital signs were unstable; blood pressure, 90/61 $\mathrm{mmHg}$; pulse rate, 50 beats/minutes, respiratory rate; 10 breaths/minutes; body temperature, $35.5^{\circ} \mathrm{C}$; and oxygen saturation, under 90\%. Endotracheal intubation was performed immediately and resuscitation was started. Initial FAST was negative. Despite of fluid resuscitation and transfusions, hypotension continued and cardiac arrest was noted. After cardiopulmonary resuscitation less than two minutes, cardiac rhythm was recovered. Since the amount of chest drain was still unnoticeable, REBOA was planned for the control of pelvic arterial bleeding. A 7 Fr Radiofocus ${ }^{\circledR}$ Introducer II (Terumo, Tokyo, Japan) was inserted via left common femoral artery, and then RESCUE balloon ${ }^{\mathrm{TM}}$
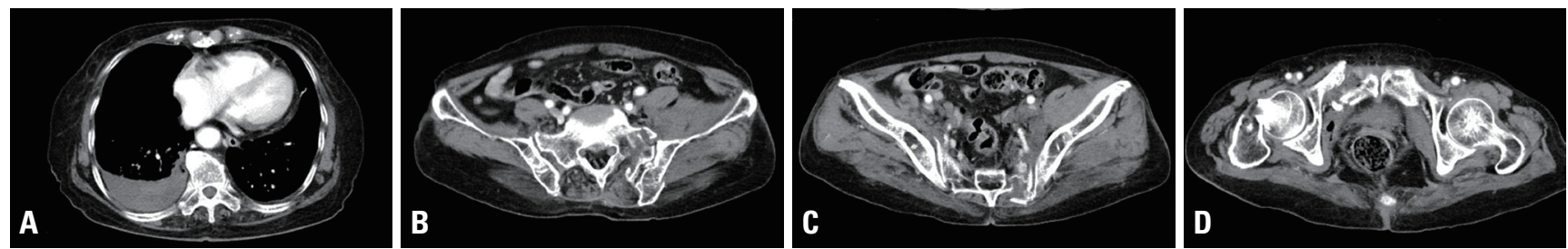

Fig. 1. (A) Computed tomography scan of right hemothorax and (B-D) unstable pelvic bone fracture with retroperitoneal hemorrhage.
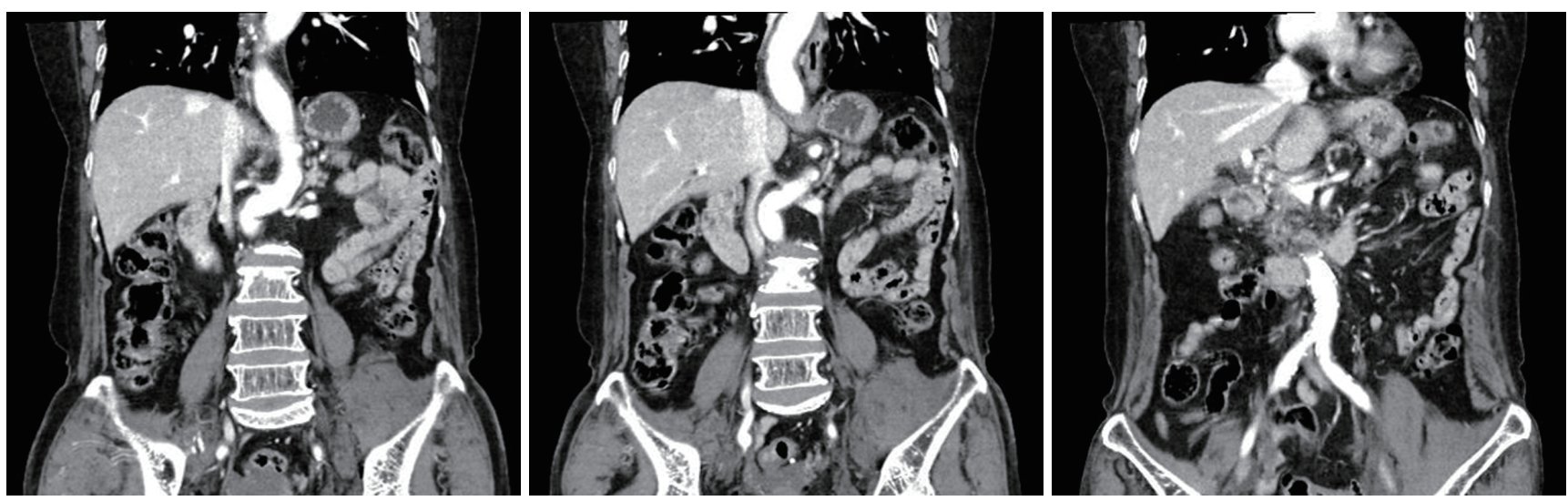

Fig. 2. Computed tomography shows tortuous aorta of the patient. Balloon reposition from Zone III to Zone I was impossible due to the anatomy. 


\section{JTI}

catheter (Tokai Medical Products, Aichi, Japan) was positioned at Zone III. The balloon was inflated to $20 \mathrm{cc}$ and blood pressure was recovered to $99 / 55 \mathrm{mmHg}$. But blood pressure was dropped to $74 / 55 \mathrm{mmHg}$ and repeated FAST showed some fluid collection in Morison pouch. Preperitoneal pelvic packing was planned for the control of pelvic venous bleeding and reposition of the REBOA balloon to Zone I was tried simultaneously. However, advancement was failed due to the tortuosity of the aorta (Fig. 2). Since a guidewire was unable to advance through the prior balloon catheter, a new REBOA balloon catheter was inserted through the same sheath and positioned at Zone I with $15 \mathrm{cc}$ inflation (Fig. 2C). Blood pressure was increased to $74 / 55 \mathrm{mmHg}$ but cardiac arrest was noted again. After the ballooning with $25 \mathrm{cc}$ (nearly total occlusion), blood pressure recovered to $101 / 76 \mathrm{mmHg}$ with a sudden gush out of blood from right chest drain. Approximately 1,000 mL of hemothorax was drained rapidly, so the balloon was repositioned at high Zone I (just below origin of left subclavian artery) (Fig. 3) and emergency department thora-

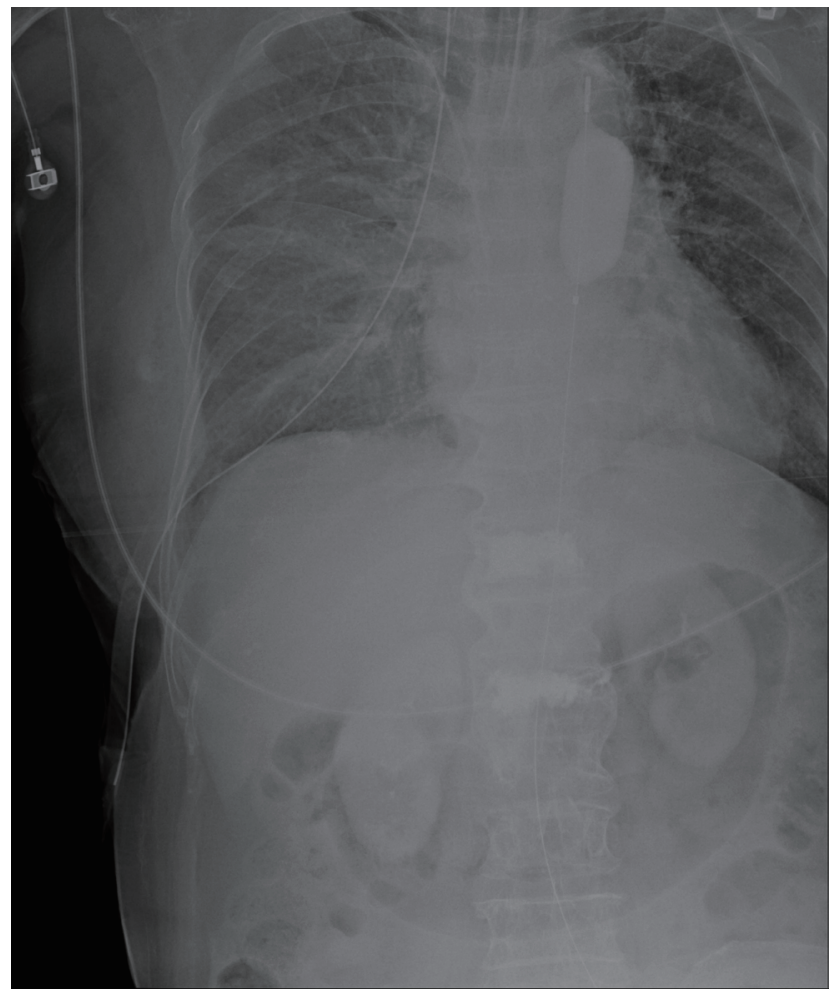

Fig. 3. The tip of resuscitative endovascular balloon occlusion of the aorta catheter is located at high Zone I. cotomy was done. There was no visible large vessel injury but multiple intercostal vessel injuries were observed near the fractured ribs. Despite the control of all sources of bleeding, the patient became coagulopathic and eventually died in the trauma bay. Three packs of universal $\mathrm{O}^{+}$ RBC were transfused within 15 minutes, and 18 packed RBCs were then transfused. Cause of death was thought to be massive hemorrhage from the right chest wall and pelvic bone fractures. The timeline of the management is shown in Table 1.

Table 1. Timeline of the management in the trauma bay

\begin{tabular}{|c|c|}
\hline $\begin{array}{l}\text { Time after arrival } \\
\text { (minutes) }\end{array}$ & $\begin{array}{l}\text { Clinical status of the patient and the } \\
\text { management }\end{array}$ \\
\hline 0 & $\begin{array}{l}\text { BP, } 90 / 61 \mathrm{mmHg} ; \mathrm{PR}, 50 / \mathrm{min} ; \mathrm{RR}, 10 / \mathrm{min} ; \mathrm{BT}, 35.5^{\circ} \mathrm{C} \\
\text { FAST (-) }\end{array}$ \\
\hline 3 & Central catheterization and fluid resuscitation \\
\hline 5 & Trauma team activation \\
\hline 6 & Endotracheal intubation \\
\hline 8 & $\begin{array}{l}\text { Cardiac arrest and CPR; 7-Fr sheath insertion via } \\
\text { the left femoral artery }\end{array}$ \\
\hline 10 & ROSC \\
\hline 14 & Zone III REBOA; 20 mL inflation; BP, 99/55 mmHg \\
\hline 16 & Repeated FAST; Morrison pouch (+) \\
\hline 20 & $\begin{array}{l}\mathrm{BP}, 74 / 55 \mathrm{mmHg} \text {; PPP was started } \\
\text { REBOA balloon repositioning from Zone III to I: } \\
\text { failed }\end{array}$ \\
\hline 23 & $\begin{array}{l}\text { Another balloon insertion at Zone l; } 15 \mathrm{~mL} \\
\text { inflation; } \mathrm{BP}, 74 / 55 \mathrm{mmHg}\end{array}$ \\
\hline 25 & Cardiac arrest and CPR; ROSC \\
\hline 26 & $\begin{array}{l}25 \mathrm{~mL} \text { inflated (nearly total occlusion); } \\
\text { BP, 101/76 mmHg }\end{array}$ \\
\hline 33 & PPP was completed \\
\hline 38 & $\begin{array}{l}\text { Exsanguination from the right chest drain } \\
(1,000 \mathrm{~mL}) \\
\text { Balloon repositioning to high Zone l; cardiac arrest } \\
\text { and CPR }\end{array}$ \\
\hline 44 & Emergency department thoracotomy \\
\hline 49 & Cardiac arrest sustained. Declared death \\
\hline
\end{tabular}

BP: blood pressure, BT: body temperature, CPR: cardiopulmonary resuscitation, FAST: focused assessment with sonography for trauma, PPP: preperitoneal pelvic packing, PR: pulse rates, REBOA: resuscitative endovascular balloon occlusion of the aorta, ROSC: return of spontaneous circulation, RR: respiratory rate. 


\section{DISCUSSION}

During the REBOA procedure, the blood flow is partially or totally blocked below the inflated balloon while the flow above is preserved. Due the change of hemodynamics, occlusion of the aorta may aggravate bleeding in sites proximal to occlusions, even in the case of minor injuries without active bleeding at the initial diagnosis [3]. In this case, there was no noticeable abdominal injury on the outside CT scan and the amount of hemothorax drained from the chest drain was minimal. Zone III REBOA was decided since main source of bleeding was pelvis which was causing a sudden cardiovascular collapse. However, as with other case reports $[3,4]$, the patient deteriorated rapidly due to the massive exsanguination from abdomen and chest after Zone III REBOA. Careful consideration of the appropriate position of the balloon is needed before the application of REBOA when there is concomitant injury proximal to the targeted zone even if it looks like minor injury. Anticipation of the adverse effect and proactive management such as repeated FAST and close monitoring of amount and nature of the chest drainage are mandatory. The possible option may be high Zone I REBOA when there are worrisome features of aggravation of the bleeding from the chest.

Second, advancement of REBOA balloon from Zone III to Zone I in a tortuous aorta is very risky. For the advancement, stiff wire needs to be withdrawn from the catheter tip and then the balloon catheter can be advanced into the aorta slowly after deflation. However, REBOA catheter may injure the wall of the aorta despite of all those efforts and the deflation during advancement may lead to catastrophic hemodynamic collapse. The advancement could eventually fail which necessitate a new or additional balloon insertion resulting in procedure time delay in patients with profound shock. We failed to advance the balloon in this patient due to the tortuosity of the aorta. In elderly patients, tortuous aorta is common and often we have to apply REBOA urgently without CT or fluoroscopic guidance. Therefore, Zone I REBOA may be preferentially considered in patients with old age or suspected/proved tortuous aorta in radiologic study.

Previous literatures suggest that Zone I REBOA was superior to Zone III in a view of hemodynamics [2,5-7].
Since Zone III REBOA may not optimally elevate proximal blood pressure in patients with severe hemorrhagic shock [8], some researchers suggest that Zone I REBOA should be considered for patients at risk for hemodynamic collapse regardless of the injury site [5]. However, Zone I REBOA may lead to an additional ischemia-reperfusion injury compared to Zone III REBOA since organ ischemia below the occlusion is inevitable $[2,7,9]$. In patients with obviously isolated and unstable pelvic fractures, risk may exceed the benefit of Zone I REBOA. Therefore, the decision should be made with caution based on the various clinical and radiologic information of the patients

In conclusion, careful consideration of the necessity of REBOA and appropriate zone selection is needed before the application when there is concomitant injury proximal to the targeted zone. High Zone I REBOA can be an option when there is combined minor chest injury. Zone I REBOA may also be preferentially performed in patients with or suspected tortuous aorta or in patients with profound shock.

\section{REFERENCES}

1. Bulger EM, Perina DG, Qasim Z, Beldowicz B, Brenner M, Guyette F, et al. Clinical use of resuscitative endovascular balloon occlusion of the aorta (REBOA) in civilian trauma systems in the USA, 2019: a joint statement from the American College of Surgeons Committee on Trauma, the American College of Emergency Physicians, the National Association of Emergency Medical Services Physicians and the National Association of Emergency Medical Technicians. Trauma Surg Acute Care Open 2019;4:e000376.

2. Kim DH, Chang SW, Matsumoto J. The utilization of resuscitative endovascular balloon occlusion of the aorta: preparation, technique, and the implementation of a novel approach to stabilizing hemorrhage. J Thorac Dis 2018;10:5550-9.

3. Maruhashi T, Minehara H, Takeuchi I, Kataoka Y, Asari Y. Resuscitative endovascular balloon occlusion of the aorta may increase the bleeding of minor thoracic injury in severe multiple trauma patients: a case report. J Med Case Rep 2017;11:347.

4. Daskal Y, Hörer T, Hebron D, Shvili Y, Kessel B. Be aware: REBOA may increase liver bleeding!. J Endovasc Resusc Trauma Manag 2018;2:68-71. 
5. Tibbits EM, Hoareau GL, Simon MA, Davidson AJ, DeSoucy ES, Faulconer ER, et al. Location is everything: the hemodynamic effects of REBOA in zone 1 versus zone 3 of the aorta. J Trauma Acute Care Surg 2018;85:101-7.

6. Beyer CA, Johnson MA, Galante JM, DuBose JJ. Zones matter: hemodynamic effects of zone 1 vs zone 3 resuscitative endovascular balloon occlusion of the aorta placement in trauma patients. Injury 2019;50:855-8.

7. Ordoñez CA, Parra MW, Manzano-Nunez R, Herrera-Escobar JP, Serna JJ, Rodriguez Ossa P, et al. Intraoperative combination of resuscitative endovascular balloon occlusion of the aorta and a median sternotomy in hemodynamically unstable patients with penetrating chest trauma: is this feasible?. J Trauma Acute Care Surg 2018;84:752-7.

8. Uchino H, Tamura N, Echigoya R, Ikegami T, Fukuoka T. "REBOA" - is it really safe? A case with massive intracranial hemorrhage possibly due to endovascular balloon occlusion of the aorta (REBOA). Am J Case Rep 2016;17:810-3.

9. Davidson AJ, Russo RM, DuBose JJ, Roberts J, Jurkovich GJ, Galante JM. Potential benefit of early operative utilization of low profile, partial resuscitative endovascular balloon occlusion of the aorta (P-REBOA) in major traumatic hemorrhage. Trauma Surg Acute Care Open 2016;1:e000028. 